\title{
On the Security of Dynamic Group Signatures: Preventing Signature Hijacking
}

\author{
Yusuke Sakai ${ }^{1, \star}$, Jacob C.N. Schuldt ${ }^{2, \star \star}$, Keita Emura ${ }^{3, \star \star \star}$, \\ Goichiro Hanaoka ${ }^{2}$, and Kazuo Ohta ${ }^{1}$ \\ 1 The University of Electro-Communications, Japan \\ yusuke.sakai@uec.ac.jp \\ 2 National Institute of Advanced Industrial Science and Technology, Japan \\ 3 National Institute of Information and Communications Technology, Japan
}

\begin{abstract}
We identify a potential weakness in the standard security model for dynamic group signatures which appears to have been overlooked previously. More specifically, we highlight that even if a scheme provably meets the security requirements of the model, a malicious group member can potentially claim ownership of a group signature produced by an honest group member by forging a proof of ownership. This property leads to a number of vulnerabilities in scenarios in which dynamic group signatures are likely to be used. We furthermore show that the currently most efficient dynamic group signature scheme does not provide protection against this type of malicious behavior.

To address this, we introduce the notion of opening soundness for group signatures which essentially requires that it is infeasible to produce a proof of ownership of a valid group signature for any user except the original signer. We then show a relatively simple modification of the scheme by Groth (ASIACRYPT 2007, full version) which allows us to prove opening soundness for the modified scheme without introducing any additional assumptions.

We believe that opening soundness is an important and natural security requirement for group signatures, and hope that future schemes will adopt this type of security.
\end{abstract}

\section{Introduction}

Group signatures, introduced by Chaum and van Heyst 11], allow a group member to anonymously sign a message on behalf of the group. More specifically, anyone will be able to verify that a signature originates from a group member, but the signature does not reveal the identity of the signer, not even to other members of the group. Group membership is controlled by an authority called the issuer, who handles enrollment of users through an interactive join protocol.

* The first author is supported by a JSPS Fellowship for Young Scientists.

$\star \star$ The second author is supported by a JSPS Fellowship for Young Scientists.

$\star \star \star$ This work was done when the third author was a postdoctoral researcher at Center for Highly Dependable Embedded Systems Technology, Japan Advanced Institute of Science and Technology (JAIST). 
To prevent misuse of the signing capabilities obtained by group members, another authority called the opener can revoke the anonymity of a signature and identify the signer of the message.

Following the introduction of group signatures, a series of different security requirements were proposed for this primitive, each of which aims at addressing a specific security concern by augmenting or refining previous notions, e.g. unforgeability, exculpability, traceability, coalition resistance, framing resistance, anonymity and unlinkability. These security notions were later consolidated in the security model proposed by Bellare, Micciancio, and Warinschi 2 who introduce two strong security requirements, full-anonymity and full-traceability, which imply all of the previously proposed notions of security.

However, a drawback of the model by Bellare, Micciancio, and Warinschi 2 is that only static group signature schemes are considered i.e. the set of group members is fixed, and the private key material of each group member is generated in the setup phase of the scheme. Furthermore, the authority controlling the group (which acts as both the issuer and opener) is considered to be fully trusted. To address this, Bellare, Shi, and Zhang [3] extended the model of [2] to capture dynamic group signature schemes in which a user can dynamically join the group by engaging in a join protocol with the issuer. Furthermore, to reduce trust in the opener, the model adopts the approach by Camenisch and Michels [10], and requires that the opener produces a non-interactive and publicly verifiable proof that a given signature was produced by a given signer. The model introduces three formal security notions: anonymity, traceability, and nonframeability. The former two notions are adaptations of the full-anonymity and full-traceability notions to the dynamic group signature setting. The latter notion, non-frameability, requires that even if a malicious opener and issuer collude, they cannot frame an honest user by producing a signature and corresponding opening which identify the honest user as the signer, when the honest user did not produce the signature in question.

Limitations of Non-frameability. While non-frameability is a strong security notion, it only partly covers the security properties one would intuitively expect to gain when the opener is required to produce a non-interactive and publicly verifiable proof of an opening. More specifically, the non-frameability notion only ensures that the opener cannot frame an uncorrupted user by constructing a proof that the user is the signer of a signature he did not produce. However, no guarantee is given regarding an opening involving a corrupted user. This leaves open the possibility that an opening showing that a malicious or corrupted user is the signer of a signature produced by an honest user, can be constructed. Furthermore, this might not require the opener to be corrupted or malicious, in which case a malicious user might be able to independently forge a proof showing that he is the signer of any signature of his choice.

Depending on the concrete scenario in which a dynamic group signature scheme is used, the ability to forge an opening proof might become a real security concern. We highlight several potential threats that this ability gives rise to: 
- Signer impersonation. The most obvious threat is signer impersonation. This is a problem if a group signature scheme is used for an anonymous auction as suggested in [1. In this scenario, the bidders correspond to group members, and when submitting a bid, a group member will attach a group signature on his bid. The opener serves as the auctioneer, and will make the opening of the signature on the highest bid public. This will enable anyone to verify who the winner of the auction is. However, a malicious bidder may forge a proof of ownership of the signature on the highest bid and may insist that he/she is the winner.

A similar situation occurs if a dynamic group signature scheme is used to implement an authentication scheme with identity escrow 23. In this case, a malicious group member can claim to be the user who authenticated himself to a server (and provide a proof thereof) when this is not the case.

- Proxy confession. The ability to open a group signature is introduced to keep the group members accountable of the messages signed on behalf of the group. However, assume that a signature on some message causes a dispute, but the real signer wants to avoid being blamed for this. Then the real signer asks (or intimidates) another group member to forge a proof of ownership of the signature and take the blame.

- Key exposure. Consider the case in which a group member's private key is exposed and falls into the hands of a malicious user. This will not only allow the malicious user to construct future signatures on any message of this choice, but will furthermore allow him to claim (and prove) that the original user is the signer of any previously generated signature.

Our Contribution. We highlight the above described potential weakness of the security guarantee provided by the formal model of Bellare, Shi, and Zhang 3 . Furthermore, we show that this is not only a property of the security model, but that the most efficient dynamic group signature schemes enable a malicious group member to forge a proof of ownership of a signature.

To address this, we propose a new security notion for dynamic group signatures which we denote opening soundness. We consider two variants of this notion, weak opening soundness and (ordinary) opening soundness. The former is intended to address the above highlighted security threats in an intuitive and straightforward manner, and will rule out the possibility that a malicious group member can produce a proof of ownership of a signature generated by an honest user. The latter considers a stronger adversary who has access to the private key of the opener, and who is only required to produce two different openings of a maliciously constructed signature. The notion of opening soundness implies the notion of weak opening soundness.

As a positive result, we prove that the generic construction of a dynamic group signature scheme by Bellare, Shi, and Zhang $[3]$ achieves opening soundness. We furthermore propose a modification of the scheme by Groth [19] which allows us to prove opening soundness of the modified scheme. In contrast, we show that 
the original scheme does not provide weak opening soundness. In addition, we briefly discuss opening soundness of the random oracle scheme [144]. A summary of our results regarding opening soundness of the above mentioned schemes can be seen in Table 1 .

Table 1. Summary of the results. The mark "?" means it is an open question whether the scheme has the given property or not. The rightmost column denotes the section in which the security of the corresponding scheme is discussed.

\begin{tabular}{|c|c|c|c|}
\hline \multicolumn{4}{|c|}{ Opening Soundness Weak Opening Soundness } \\
\hline Our Variant of 19 & YES & YES & $(\$ 5.1)$ \\
\hline Bellare-Shi-Zhang 3] & YES & YES & (4) \\
\hline Furukawa-Imai 14 & No & $?$ & (4) \\
\hline Bichsel et al. 4 & $\mathrm{No}$ & $?$ & $(\sqrt{4})$ \\
\hline Groth (full version) 19 & No & No & (94) \\
\hline
\end{tabular}

Related Work. Since the first proposal of group signature by Chaum and van Heyst, many efficient constructions have been proposed, most of which are relying on the random oracle model [16 9|2214 12 4]. Many initial schemes were based on the strong-RSA assumption. The first group signature schemes based on assumptions of the discrete-logarithm type were achieved independently by Camenisch and Lysyanskaya [9], and Boneh, Boyen, and Shacham [6]. The former scheme is based on the LRSW assumption, while the latter is based on the $q$-strong Diffie-Hellman assumption. Kiayias, Tsiounis, and Yung proposed the notion of traceable signature [21, which can be seen as an extension of group signature with additional anonymity-revocation functionalities. One of these functionalities is that of allowing a group member to claim the authorship of a signature, however, its security requirement does not care about the possibility in which a malicious member falsely claims the authorship of an honestly generated signature by another.

Constructions which are provably secure without random oracles were only recently achieved. Besides the generic construction relying on NIZK proofs for general NP languages, Groth constructed the first concrete group signature scheme with constant signature size by exploiting the properties of bilinear groups [17, though signatures are extremely large. Boyen and Waters proposed group signature schemes $7 / 8$ ] whose signature sizes are quite compact. In particular the latter scheme has signatures consisting only of six group elements of a composite order group. The drawback of these schemes is that they only achieve weaker security guarantees, that is, they only provide so called CPA-anonymity in the security model of Bellare, Micciancio, and Warinschi [2]. Groth proposed another group signature scheme 1819] which has constant signature size (roughly one or two kilobytes) and which is provably secure in the dynamic group signature model of Bellare, Shi, and Zhang [3] without relying on random oracles. 


\section{Preliminaries}

\subsection{Group Signatures}

In this section, we briefly review the model and the security notions of group signatures, presented by Bellare, Shi, and Zhang 3. A group signature scheme consists of the following seven algorithms:

GKg: This is a group key generation algorithm which, on input $1^{k}$, returns the keys $(g p k, i k, o k)$, where $g p k$ is a group public key, $i k$ is an issuing key, and $o k$ is an opening key.

UKg: This is a user key generation algorithm which, on input $g p k$, returns a personal public and private key pair $(u p k, u s k)$. Each user $i$ will generate a personal key pair $\left(u p k_{i}, u s k_{i}\right)$ before engaging in the joining protocol which is described below.

Join/Issue: This is a pair of interactive algorithms which implement the joining protocol run by a user $i$ and the issuer. The algorithm Join, which is run by the user, takes $(g p k, u p k, u s k)$ as input, whereas Issue, which is run by the issuer, takes $(g p k, u p k, i k)$ as input. Upon successful completion of the protocol, Join outputs a private signing key $g s k_{i}$ for user $i$, and Issue outputs the registration information of user $i$ which is stored in reg[i], where reg is a registration table maintained by the issuer.

GSig: This is the group signing algorithm run by a user $i$, which, on input gpk, a signing key $g s k_{i}$, and a message $m$, returns a group signature $\Sigma$.

GVf: This is the group signature verification algorithm which, on input ( $g p k, m$, $\Sigma)$, returns 1 to indicate that $\Sigma$ is a valid signature on $m$, or 0 otherwise.

Open: This is the opening algorithm run by the opener, which, on input (gpk, ok, reg, $m, \Sigma)$, returns $(i, \tau)$, where $i$ specifies that the originator of the signature $\Sigma$ is the user $i$, and $\tau$ is a non-interactive proof of this. In case the algorithm fails to identify the originator of the signature, it outputs $i=0$. Note that Open requires access to the registration table reg.

Judge: This is the judge algorithm which, on input $\left(g p k, i, u p k_{i}, m, \Sigma, \tau\right)$, outputs either 1 or 0 indicating that the proof $\tau$ is accepted as valid or invalid, respectively.

The model in [3] introduces four requirements for a group signature scheme, namely, correctness, anonymity, non-frameability, and traceability. The correctness notion requires that honestly generated signatures will be accepted as valid by the verification algorithm, can be opened by the opening algorithm, and that the judging algorithm will accept the resulting proof as valid. The anonymity notion requires that no information about the identity of a signer is leaked from a group signature, even if the signing keys of all group members and the issuer are exposed. The non-frameability notion requires that no adversary corrupting both the opener and the issuer, can produce a signature and an opening proof that identify an uncorrupted group member as the signer, when the uncorrupted group member did not produce the signature in question. The traceability notion requires that an adversary corrupting the opener and controlling a group 
of malicious group members, cannot produce a valid signature that cannot be opened correctly.

The formal definitions of the four notions are given as follows. We first define several oracles needed for security notions:

$\operatorname{AddU}(i)$ : The add-user oracle runs $\operatorname{UKg}(g p k)$ and Join/Issue protocol to add an honest user. It returns the user public key $u p k$ of the user. The oracle add $i$ to the set HU.

$\operatorname{RReg}(i)$ : The read-registration-table oracle reveals the content of the registration table reg $[i]$.

SndToU $(i, M)$ The send-to-user oracle at first sets up a user public/secret key pair by $\left(u p k_{i}, u s k_{i}\right) \leftarrow \operatorname{UKg}(g p k)$ and add $i$ to the set HU. The oracle then allows the adversary to engage a group-joining protocol of the user $i$ on the behalf of the corrupted issuer. The message $M$ is sent to the user $i$ who follows the protocol Join $\left(g p k, u p k_{i}, u s k_{i}\right)$. The response of the user is returned to the adversary.

WReg $(i, M)$ The write-registration-table oracle updates reg $[i]$ to $M$.

USK $(i)$ : The user-secret-keys oracle reveals the secret keys $\left(u s k_{i}, g s k_{i}\right)$ of the user $i$ to the adversary.

$\operatorname{CrptU}(i, M)$ : The corrupt-user oracle sets the user public key of the user $i$ to $M$ and add $i$ to the set CU.

Open $(m, \Sigma)$ : The open oracle returns the opening $(i, \tau) \leftarrow$ Open $(g p k, o k, m, \Sigma)$ of the signature $\Sigma$ under the message $m$.

$\mathrm{Ch}_{b}\left(m, i_{0}, i_{1}\right)$ : The challenge oracle returns a challenge $\Sigma^{*} \leftarrow \operatorname{GSig}\left(g p k, g s k_{i_{b}}\right.$, $m)$. The users $i_{0}$ and $i_{1}$ needs to be in the set $\mathrm{HU}$.

$\operatorname{GSig}(i, m)$ : The signing oracle returns a signature $\Sigma \leftarrow \operatorname{GSig}\left(g p k, g_{s} k_{i}, m\right)$ on the message $m$ of the user $i$, who needs to be in the set HU.

SndTol $(i, M)$ : The send-to-issuer oracle allows the adversary to engage a groupjoining protocol on behalf of the corrupted user $i$. The message $M$ is sent to the issuer who follows the protocol Issue $\left(g p k, u p k_{i}, i k\right)$. The response of the issuer is returned to the adversary. The user $i$ needs to be in the set CU.

The correctness and security requirements for a group signature scheme are as follows:

Definition 1. A group signature scheme is said to have correctness if

$$
\begin{aligned}
& \operatorname{Pr}\left[(g p k, i k, o k) \leftarrow \operatorname{GKg}\left(1^{k}\right) ;(i, m) \leftarrow \mathcal{A}^{\mathrm{AddU}, \operatorname{RReg}}(g p k)\right. \\
& \Sigma \leftarrow \operatorname{GSig}\left(g p k, g s k_{i}, m\right) ;(j, \tau) \leftarrow \operatorname{Open}(g p k, o k, r e g, m, \Sigma) \\
&\left.\quad: \operatorname{GVf}(g p k, m, \Sigma)=0 \vee i \neq j \vee \operatorname{Judge}\left(g p k, i, u p k_{i}, m, \Sigma, \tau\right)=0\right]
\end{aligned}
$$

is negligible for any probabilistic polynomial-time adversary $\mathcal{A}$.

Definition 2. A group signature scheme is said to have anonymity if

$$
\begin{aligned}
\operatorname{Pr}\left[b \leftarrow\{0,1\} ;(g p k, i k, o k) \leftarrow \operatorname{GKg}\left(1^{k}\right) ;\right. \\
\left.b^{\prime} \leftarrow \mathcal{A}^{\text {SndToU,WReg,USK,CrptU,Open,Ch }}(g p k, i k): b=b^{\prime}\right]-\frac{1}{2}
\end{aligned}
$$

is negligible for any probabilistic polynomial-time adversary $\mathcal{A}$. 
Definition 3. A group signature scheme is said to have non-frameability if

$$
\begin{aligned}
& \operatorname{Pr}\left[(g p k, i k, o k) \leftarrow \operatorname{GKg}\left(1^{k}\right) ;\right. \\
&(m, \Sigma, i, \tau) \leftarrow \mathcal{A}^{\text {SndToU,WReg,USK,CrptU,GSig }}(g p k, o k, i k) ; \\
&: \operatorname{GVf}(g p k, m, \Sigma)=1 \wedge \text { Judge }\left(g p k, i, \text { upk } k_{i}, m, \Sigma, \tau\right)=1 \\
&\wedge \mathcal{A} \text { queried neither } \operatorname{USK}(i) \text { nor } \operatorname{GSig}(i, m)]
\end{aligned}
$$

is negligible for any probabilistic polynomial-time adversary $\mathcal{A}$.

Definition 4. A group signature scheme is said to have traceability if

$$
\begin{gathered}
\operatorname{Pr}\left[(g p k, i k, o k) \leftarrow \operatorname{GKg}\left(1^{k}\right) ;(m, \Sigma) \leftarrow \mathcal{A}^{\mathrm{CrptU}, \text { SndTol,AddU,USK,RReg }}(g p k, o k) ;\right. \\
(i, \tau) \leftarrow \text { Open }(g p k, o k, r e g, m, \Sigma) \\
\left.: \operatorname{GVf}(g p k, m, \Sigma)=1 \wedge\left(i=0 \vee \operatorname{Judge}\left(g p k, i, u p k_{i}, m, \Sigma, \tau\right)=0\right)\right]
\end{gathered}
$$

is negligible for any probabilistic polynomial-time adversary $\mathcal{A}$.

\subsection{Other Primitives}

Public-Key Encryption. A public key encryption scheme consists of three algorithms (EKg, Enc, Dec), which satisfy the following correctness condition: For any security parameter $\ell \in \mathbb{N}$, any plaintext $m \in\{0,1\}^{*}$, any random tape $r$ for $\mathrm{EKg}$, and any random tape $s$ for Enc, the condition $\operatorname{Dec}(d k, \operatorname{Enc}(p k, m ; s))=m$ holds, where $p k$ and $d k$ are output from EKg as $(p k, d k) \leftarrow \operatorname{EKg}\left(1^{\ell} ; r\right)$. In this paper we require a public key encryption scheme to satisfy the security notion of indistinguishability under chosen-ciphertext attack (IND-CCA) [25].

Digital Signature. A digital signature scheme consists of three algorithms (SKg, Sign, Ver), which satisfy the following correctness condition: For any security parameter $\ell \in \mathbb{N}$, any message $m \in\{0,1\}^{*}$, any random tape $r$ for SKg, and any random tape $s$ for Sign, the condition $\operatorname{Ver}(v k, m, \operatorname{Sign}(s k, m ; s))=\top$ holds, where $v k$ and $s k$ are output from SKg as $(v k, s k) \leftarrow \operatorname{SKg}\left(1^{\ell} ; r\right)$. In this paper we use two types of security for digital signature schemes. One is the standard security notion of unforgeability under adaptive chosen message attack (EUF-CMA), and the other is strong one-time signatures. See [16] for exact definitions.

Target Collision-Resistant Hash Functions. A family of functions is called target collision-resistant if no algorithms, which firstly chooses an input and then is given a description of a function in the family, can find another input that produces the same output to the first input. The formal definition we need is as follows: A function generator HashGen $\left(1^{\ell}\right)$ takes as input a security parameter and outputs a function $\mathcal{H}$. The family of functions is said to be target collisionresistant when $\operatorname{Pr}\left[(x, s) \leftarrow \mathcal{A} ; \mathcal{H} \leftarrow \operatorname{HashGen}\left(1^{\ell}\right) ; x^{\prime} \leftarrow \mathcal{A}(\mathcal{H}, s): \mathcal{H}(x)=\mathcal{H}\left(x^{\prime}\right) \wedge\right.$ $\left.x \neq x^{\prime}\right]$ is negligible for any polynomial-time algorithm $\mathcal{A}$. 
Non-interactive Proofs. A non-interactive proof system for an NP-relation $R \in\{0,1\}^{*} \times\{0,1\}^{*}$ defining $L=\{x \mid(x, w) \in R$ for some $w\}$ consists of three algorithms $(K, P, V)$, which satisfy the following correctness and soundness conditions: For correctness, it is required that for any security parameter $\ell \in \mathbb{N}$, any common reference string crs $\leftarrow K\left(1^{\ell}\right)$, and any pair $(x, w) \in R$, it holds that $V\left(1^{\ell}, c r s, x, P\left(1^{\ell}, c r s, x, w\right)\right)=\top$; for soundness, it is required that for any $\ell \in \mathbb{N}$ and any probabilistic polynomial-time algorithm $\mathcal{A}$, the probability $\operatorname{Pr}\left[\right.$ crs $\leftarrow K\left(1^{\ell}\right) ;(x, \pi) \leftarrow \mathcal{A}\left(1^{\ell}\right.$, crs $): V\left(1^{\ell}\right.$, crs $\left.\left., x, \pi\right)=\top \wedge x \notin L\right]$ is negligible. In fact we will later use two types of proof systems, one which is zero-knowledge [5]13] and one which is simulation-sound [26] in addition to zeroknowledge.

Bilinear Maps and Groth-Sahai Proofs. Bilinear groups are groups $\mathbb{G}$ and $\mathbb{G}_{T}$ with the same order that have an efficiently computable bilinear map $e$ : $\mathbb{G} \times \mathbb{G} \rightarrow \mathbb{G}_{T}$. Let $\mathcal{G}$ be a probabilistic polynomial-time algorithm that outputs a group parameter $g k=\left(p, \mathbb{G}, \mathbb{G}_{T}, e, g\right)$ where $p$ is the order of $\mathbb{G}$ and $\mathbb{G}_{T}, e$ is a non-degenerates bilinear map $e: \mathbb{G} \times \mathbb{G} \rightarrow \mathbb{G}_{T}$, and $g$ is a generator of $\mathbb{G}$.

Groth and Sahai 20] introduced a framework for very efficient non-interactive proof for the satisfiability of some algebraic equations they called quadratic equations. The proof system consists of algorithms $\left(K_{\mathrm{NI}}, P, V, X\right)$. The algorithm $K_{\mathrm{NI}}(g k)$ takes a group parameter $g k$ as input and outputs (crs, $x k$ ), where $c r s$ is a common reference string and $x k$ is a trapdoor extraction key for extracting a witness from a proof. The algorithm $P(c r s, x, w)$ outputs a proof $\pi$ for an equation described by $x$ whose witness is $w$. A proof $\pi$ is verified by running $V(\operatorname{crs}, x, \pi)$. The algorithm $X_{x k}(x, \pi)$ extracts a witness from the proof $\pi$ which passes the verification algorithm. In fact there are two types of proof systems $\left(K_{\mathrm{NI}}, P_{\mathrm{NIWI}}, V_{\mathrm{NIWI}}, X\right)$ and $\left(K_{\mathrm{NI}}, P_{\mathrm{NIZK}}, V_{\mathrm{NIZK}}, X\right)$, which respectively provide witness-indistinguishability and zero-knowledge properties. The two proof systems have the identical common reference string generation algorithm. Moreover they share single string for different sets of equations in the Groth group signature scheme.

The common reference string consists of eight group elements as $\mathrm{crs}=$ $\left(F, H, U, V, W, U^{\prime}, V^{\prime}, W^{\prime}\right)$. A notable property on this is that $F$ and $H$ essentially serve as a public key of the linear encryption [6. This property is exploited in the Groth group signature scheme (and so in our modification of that scheme). For further details see 20 .

Tag-Based Encryption. Tag-based encryption is an extension of public key encryption, which associates an additional "tag" with a ciphertext. The exact syntax is as follows: A key generation algorithm $G\left(1^{\ell}\right)$ generates a public key $p k$ and a secret key $d k$; an encryption algorithm $E_{p k}(t, m)$ takes as input a public key $p k$, a tag $t$, and a plaintext $m$, and outputs a ciphertext $c$; a decryption algorithm $D_{d k}(t, c)$ takes as input a decryption key $d k$, a tag $t$, and a ciphertext $c$ and outputs a plaintext $m$ or a special symbol $\perp$ indicating the decryption failed. The correctness condition only ensures that the plaintext is recovered when the tags used in the encryption and the decryption are identical. 
In this paper we use Kiltz's construction of tag-based encryption [24], which is explained below. The scheme can be built on bilinear groups. Let $g k=$ $\left(p, \mathbb{G}, \mathbb{G}_{T}, e, g\right)$ be a group description. The key generation algorithm chooses random integers $\phi, \eta \leftarrow \mathbb{Z}_{p}$ and random elements $K, L \leftarrow \mathbb{G}$, and sets $p k=$ $(F, H, K, L)$ where $F=g^{\phi}$ and $H=g^{\eta}$ and $d k=(\phi, \eta)$. A ciphertext of a plaintext $m$ under a tag $t$ is computed as $y=\left(y_{1}, y_{2}, y_{3}, y_{4}, y_{5}\right)=\left(F^{r}, H^{s}, m g^{r+s}\right.$, $\left.\left(g^{t} K\right)^{r},\left(g^{t} L\right)^{s}\right)$. The decryption algorithm decrypts a ciphertext $\left(y_{1}, y_{2}, y_{3}, y_{4}\right.$, $\left.y_{5}\right)$ under a tag $t$ by checking $e\left(F, y_{4}\right)=e\left(y_{1}, g^{t} K\right)$ and $e\left(H, y_{5}\right)=e\left(y_{2}, g^{t} L\right)$ and outputs $y_{3} / y_{1}^{\phi} y_{2}^{\eta}$ if the two equations hold, otherwise outputs $\perp$. This encryption scheme is secure against selective-tag weak chosen-ciphertext attacks if the decisional linear assumption holds 24. Another interesting property is that the scheme has public verifiability in the sense that it can be efficiently checked whether a given five-tuple $\left(y_{1}, y_{2}, y_{3}, y_{4}, y_{5}\right)$ lies in the range of the encryption algorithm under a given public key $p k$ and a given tag $t$ by checking the two equations $e\left(F, y_{4}\right)=e\left(y_{1}, g^{t} K\right)$ and $e\left(H, y_{5}\right)=e\left(y_{2}, g^{t} L\right)$.

\section{Opening Soundness}

In this section we give a formal definition of opening soundness. Specifically, we introduce two variants of opening soundness, weaker and stronger definitions.

The weaker definition, named weak opening soundness, is intended to address the security concerns discussed in the introduction in a straightforward manner, and will rule out the possibility that a malicious user can claim ownership of a signature produced by an honest user by forging an opening proof. The definition is as follows:

Definition 5. A group signature scheme is said to have weak opening soundness if

$$
\begin{aligned}
& \operatorname{Pr}\left[(g p k, i k, o k) \leftarrow \operatorname{GKg}\left(1^{k}\right) ;\left(m, i, i^{*}, s\right) \leftarrow \mathcal{A}^{\operatorname{AddU}(\cdot)}(g p k)\right. \\
& \Sigma \leftarrow \operatorname{GSig}\left(g p k, g s k_{i}, m\right) ; \tau^{*} \leftarrow \mathcal{A}^{\operatorname{AddU}(\cdot)}\left(s, \Sigma, g s k_{i^{*}}\right) \\
&\left.\quad: i \neq i^{*} \wedge i, i^{*} \in \mathrm{HU} \wedge \operatorname{Judge}\left(g p k, u p k_{i^{*}}, m, \Sigma, \tau^{*}\right)=1\right]
\end{aligned}
$$

is negligible for all polynomial time adversaries $\mathcal{A}$, where the oracle $\mathrm{AddU}$ is defined as follows:

AddU: On a query $i \in \mathbb{N}$, the oracle runs $\left(u p k_{i}, u s k_{i}\right) \leftarrow \operatorname{UKg}(g p k)$, then executes the protocol $\left(g_{s} k_{i}, r_{e} g_{i}\right) \leftarrow\left\langle J_{o i n}\left(g p k, u p k_{i}, u s k_{i}\right)\right.$, Issue $\left.(g p k, i k)\right\rangle$, adds $i$ to a set $\mathcal{H} \mathcal{U}$, and lastly returns $u p k_{i}$.

Note that the adversary is only allowed to receive the secret signing key of a single user $i^{*}$. Hence, this definition will not rule out attacks involving a corrupted opener, and therefore cannot contribute towards reducing trust in this entity.

In contrast, the stronger definition, named opening soundness, is intended to rule out the possibility that an adversary can produce two different openings of a signature, even if he is allowed to corrupt the opener and all the users in the system, and furthermore generate the signature in question maliciously. The definition is as follows: 
Definition 6. A group signature scheme is said to have opening soundness if

$$
\begin{gathered}
\operatorname{Pr}\left[(g p k, i k, o k) \leftarrow \operatorname{GKg}\left(1^{k}\right) ;\left(m, \Sigma, i_{1}, \tau_{1}, i_{2}, \tau_{2}\right) \leftarrow \mathcal{A}^{\mathrm{CrptU}, \mathrm{WReg}}(g p k, o k, i k)\right. \\
: \operatorname{GVf}(g p k, m, \Sigma)=1 \wedge i_{1} \neq i_{2} \wedge \operatorname{Judge}\left(g p k, u p k_{i_{1}}, m, \Sigma, \tau_{1}\right)=1 \\
\left.\wedge \operatorname{Judge}\left(g p k, u p k_{i_{2}}, m, \Sigma, \tau_{2}\right)=1\right]
\end{gathered}
$$

is negligible for all polynomial time adversaries $\mathcal{A}$, where the oracle $\operatorname{CrptU}(i, M)$ sets the user public key of the user $i$ to be $M$, and the oracle $\operatorname{WReg}(i, M)$ sets reg[i] to $M$.

While the weaker definition provides a minimum level of protection against the type of attacks described in the introduction, we believe that, when applied to the scenarios mentioned in the introduction, any dynamic group signature scheme should provide (ordinary) opening soundness to prevent any type of attack which exploits ambiguity of openings, or involves a corrupted opener. Furthermore, we will show that this level of security can be achieved efficiently by showing that our modified version of the scheme by Groth provides opening soundness (See Sect. 5 for details).

\section{Opening Soundness of Existing Schemes}

We will now take a closer look at some of the existing dynamic group signature schemes, and highlight the level of opening soundness (ordinary, weak or none) achieved by these. Note that since the Bellare-Shi-Zhang security model for dynamic group signatures does not considers opening soundness, a security proof in this model will not allow us to make any conclusions regarding the opening soundness of existing schemes.

In this section, we will focus on the standard model scheme by Groth described in [19] (note that the updated scheme in [19] is slightly different from the scheme described in [18]) and the generic construction of a dynamic group signature scheme by Bellare, Shi, and Zhang [3. More specifically, we will show that the scheme by Groth does not have weak opening soundness whereas the generic construction by Bellare, Shi and Zhang has opening soundness. We further show that the random oracle model schemes by Furukawa and Imai [14] and Bichsel et al. 4] do not have opening soundness. Interestingly, while these schemes do not provide opening soundness, there seems to be no obvious attack against the weak opening soundness of these.

The Groth Scheme. Figure1 1shows a description of the Groth scheme. Below, we will expand on the description given in the figure.

In the group key generation algorithm $\mathrm{GKg}$, the elements $f, h, T$ correspond to a verification key of the Zhou-Lin signature scheme [27], whereas $z$ corresponds to a signing key. Furthermore, $p k$ is a public key of Kiltz's tag-based encryption scheme. Note that the first two elements of $p k$ and the common reference string crs for the non-interactive Groth-Sahai proofs are identical. 


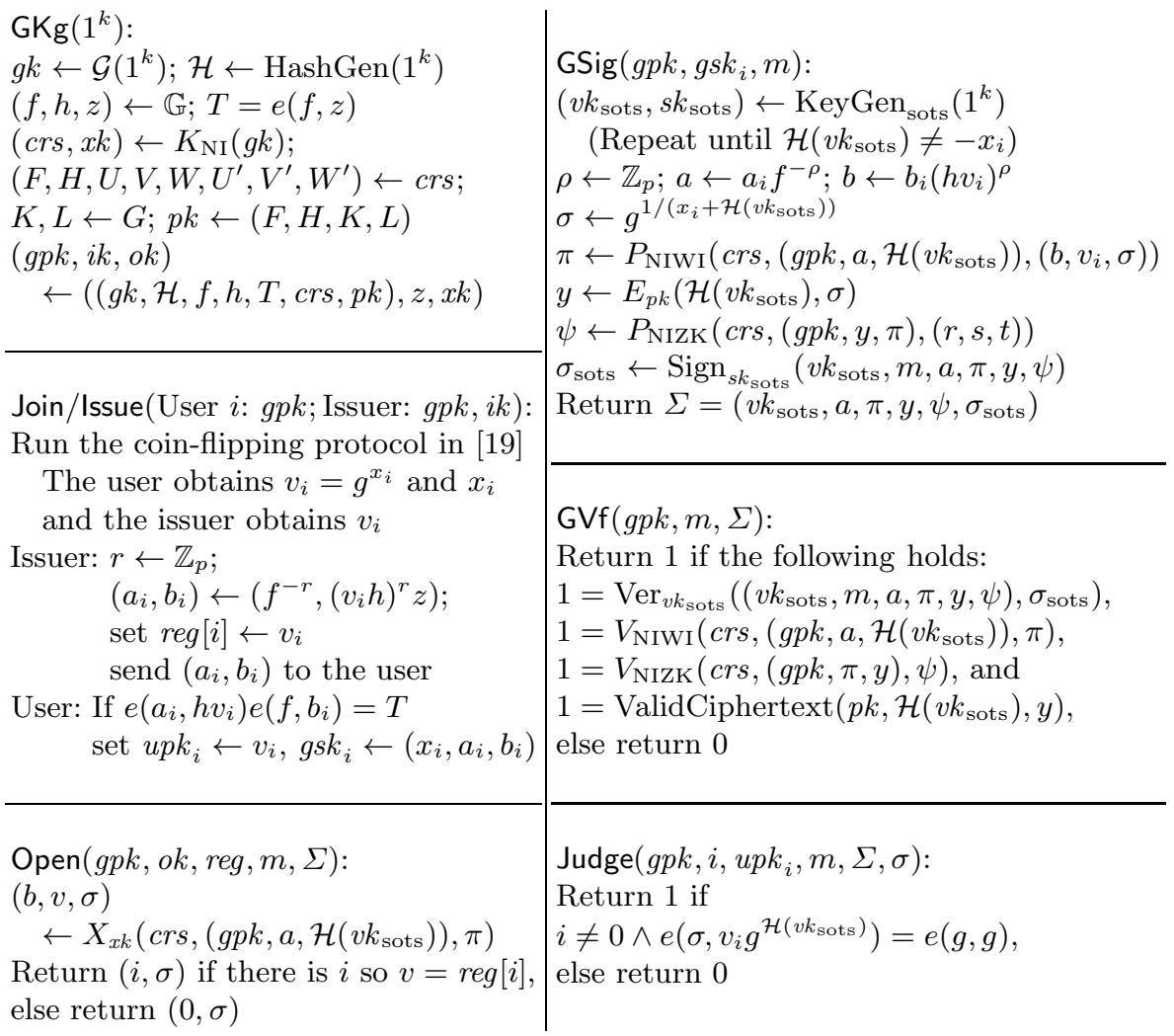

Fig. 1. The Groth group signature scheme [19]

In the group signing algorithm GSig, a group member constructs two noninteractive Groth-Sahai proofs. The first proof $\pi$, constructed via $P_{\text {NIWI }}$, shows knowledge of a signature $\sigma$, a verification key $v$ and a part $b$ of a (re-randomized) certificate $(a, b)$ which satisfy $e(a, h v) e(f, b)=T \wedge e\left(\sigma, v g^{\mathcal{H}\left(v k_{\text {sots }}\right)}\right)=e(g, g)$. The first part $a$ of the certificate can safely be revealed as part of the group signature since it does not leak any information about the identity of the member due to the re-randomization. The second proof $\psi$, constructed via $P_{\text {NIZK }}$, demonstrates that the plaintext of $y$ is the same as the witness $\sigma$ used in $\pi$. Let us explain in detailed. The tag-based encryption $y$ has the form $\left(y_{1}, y_{2}, y_{3}, y_{4}, y_{5}\right)=$ $\left(F^{r_{y}}, H^{s_{y}}, g^{r_{y}+s_{y}} \sigma,\left(g^{\mathcal{H}\left(v k_{\mathrm{sots}}\right)} K\right)^{r_{y}},\left(g^{\mathcal{H}\left(v k_{\mathrm{sots}}\right)} L\right)^{s_{y}}\right)$, while the Groth-Sahai proof $\pi$ contains a commitment $c=\left(c_{1}, c_{2}, c_{3}\right)=\left(F^{r_{c}} U^{t}, H^{s_{c}} V^{t}, g^{r_{c}+s_{c}} W^{t} \sigma\right)$. The proof demonstrates that there exists $(r, s, t)$ such that $\left(c_{1} y_{1}^{-1}, c_{2} y_{2}^{-1}, c_{3} y_{3}^{-1}\right)=$ $\left(F^{r} U^{t}, H^{s} V^{t}, g^{r+s} W^{t}\right)$. When $y$ and $c$ encrypt the same message, there exists $(r, s, t)$ that satisfies above equation, but if $y$ and $c$ encrypt different messages, no such tuple $(r, s, t)$ exists.

The verification algorithm GVf will, in addition to the verification of the two non-interactive proofs and the one-time signature, verify that the ciphertext $y$ 
is a valid ciphertext, using the algorithm ValidCiphertext. This algorithm is easily implemented for the tag-based encryption scheme by Kiltz (see the last paragraph of Sect. 2 for details).

We will now show how a malicious group member can forge a opening proof which shows that he is the signer of any signature $\Sigma$ produced by user $i$. As shown above, an opening proof consists of a certified signature $\sigma$ on $v k_{\text {sots }}$ which is part of $\Sigma$. To verify the opening proof, it is only verified that $\sigma$ is a valid signature on $v k_{\text {sots }}$ under the verification key $v_{i}$ of the user in question.

Hence, a malicious user $i^{\prime}$ who wants to impersonate the signer of the group signature $\Sigma$ on $m$, simply uses his own private signing $x_{i^{\prime}}$ key to construct a new signature $\sigma^{\prime}$ on $v k_{\text {sots }}$, and publicizes this as an opening proof together with his own identity $i^{\prime}$. This proof will be accepted by the Judge algorithm since $\sigma^{\prime}$ is a valid signature in $v k_{\text {sots }}$.

We formally state this as a theorem:

Theorem 1. The Groth group signature scheme does not provide weak opening soundness.

Proof. We describe an algorithm for producing a forged proof: When the adversary receives the security parameter $1^{\ell}$ and a group public key $g p k$, it firstly issues two queries $\operatorname{AddU}(1)$ and $\operatorname{AddU}(2)$ in order to add two members 1 and 2 the group. The adversary then requests the challenge by outputting $\left(i, i^{*}, m\right)=$ $\left(1,2,0^{\ell}\right)$, and receives a tuple $\left(\Sigma, g s k_{2}\right)$, where $\Sigma=\left(v k_{\text {sots }}, a, \pi, y, \psi, \sigma_{\text {sots }}\right)$ and $g s k_{2}=\left(x_{2}, a_{2}, b_{2}\right)$. The adversary forges a proof of ownership by computing $\sigma^{*}=g^{1 /\left(x_{2}+\mathcal{H}\left(v k_{\mathrm{sots}}\right)\right)}$ and outputs $\sigma^{*}$ (Notice that $v k_{\text {sots }}$ is taken from the group signature $\Sigma$ ).

One can easily verify that Judge $\left(g p k, 2, \operatorname{reg}[2], m, \Sigma, \sigma^{*}\right)$ actually outputs 1 , which means that the algorithm successfully breaks the opening soundness.

The Bellare-Shi-Zhang Scheme. Below, we will give an intuitive description of the generic construction of a dynamic group signature scheme by Bellare, Shi, and Zhang.

In the Bellare-Shi-Zhang construction, each group member $i$ has a key pair $\left(v k_{i}, s k_{i}\right)$ of an EUF-CMA secure signature scheme. The issuer also possesses his own key pair $(a k, c k)$ of the signature scheme. The issuer signs the message $\left\langle i, v k_{i}\right\rangle$ to obtain the signature cert $_{i}$, and sends cert ${ }_{i}$ to the user $i$. A group signature on a message $m$ by the user $i$ is a pair $(C, \pi)$ : here $C$ is an encryption of $\left\langle i, v k_{i}, \operatorname{cert}_{i}, s\right\rangle, s$ is a signature on $m$ under the key pair $\left(v k_{i}, s k_{i}\right)$, and the NIZK proof $\pi$ proves that the plaintext encrypted in $C$ is of the form $\langle i, v k$, cert, $s\rangle$. The opener attributes a group signature $\Sigma=(C, \pi)$ to the user $i$ by providing an NIZK proof $\tau$ for another statement (i.e. different from that of $\pi$ ), which claims the existence of a decryption key that corresponds to the opener's public key and that under that key $C$ is decrypted to $\left\langle i, v k_{i}, c e r t_{i}, s\right\rangle$.

This simple scheme provides opening soundness. Intuitively, this is due to the correctness of the public key encryption used to encrypt the signature and the certificate, and the soundness of the NIZK proof system for $\tau$. The correctness 
condition of public key encryption ensures that given a public key $p k$ and a ciphertext $C$, the decryption of $C$ is determined uniquely. Now, let us assume that an adversary of the opening soundness game outputs a tuple $\left(m, \Sigma, i_{1}, \tau_{1}, i_{2}, \tau_{2}\right)$ where $\Sigma=(C, \pi)$ and wins the game. The proof $\tau_{1}$ proves that $C$ decrypts to $\left\langle i_{1}, v k\right.$, cert, $\left.s\right\rangle$ for some $v k$, cert, and $s$, whereas $\tau_{2}$ proves that $C$ decrypts to a different plaintext $\left\langle i_{2}, v k^{\prime}, c e r t^{\prime}, s^{\prime}\right\rangle$ for some $v k^{\prime}, c e r t^{\prime}$, and $s^{\prime}$. However, this should not be possible since the decryption of $C$ under a fixed public key is unique. Hence, the adversary breaks the soundness of the NIZK proof system.

A formal statement and its proof are deferred to the full version.

The Furukawa-Imai Scheme. The Furukawa-Imai group signature scheme does not have opening soundness, which we will show in the following.

The scheme exploits a group $\mathbb{G}$ on which the decisional Diffie-Hellman assumption holds, in addition to bilinear groups $\left(\mathbb{G}_{1}, \mathbb{G}_{2}, \mathbb{G}_{T}\right)$ with an asymmetric bilinear map $e: \mathbb{G}_{1} \times \mathbb{G}_{2} \rightarrow \mathbb{G}_{T}$. In this scheme, each group member $i$ has a public key $Q_{i}=g^{x_{i}}$ and its corresponding secret key $x_{i}$. The public key $Q_{i}$ is encrypted in a group signature with (a kind of) ElGamal encryption. Let $(R, V)=\left(Q_{i} g^{r}, S^{r}\right)$ be the ciphertext that appears in a group signature, where $S=g^{s}$ is the public key of the ElGamal encryption. The opener possesses the decryption key $s$, and identifies the signer by decrypting the ciphertext. An opening contains a proof of knowledge of $w$ such that $Q_{i}=R / V^{1 / w}$, where $Q_{i}$ is the public key of the specified member (The opener uses $s$ as the witness for the above equation).

If the adversary corrupts the opener and two different members $i$ and $j$, the adversary can construct two different openings of a single signature, each of which attributes the signature to the user $i$ or the user $j$, respectively. The adversary proceeds as follows: At first the signature is honestly generated by the user $i$. Let $(R, V)=\left(g^{x_{i}+r}, S^{r}\right)$ be the ciphertext contained in this signature. The first opening is also honestly generated by the opener to attribute the signature to $i$. The second proof is generated by computing a proof of knowledge $w$ that satisfies $Q_{j}=R / V^{1 / w}$ with the witness $w=s r /\left(x_{i}+r-x_{j}\right)$. This proof attributes the signature to the user $j$. Note that the randomness $r$ for the encryption is reused to forge the second proof. This is the reason why the adversary needs to corrupt the user $i$, not only the user $j$ and the opener.

The Bichsel et al. Scheme. In the Bichsel et al. scheme, a group member receives a Camenisch-Lysyanskaya signature on a random message $\xi$ from the issuer. To generate a group signature, the member rerandomizes the certificate and computes a "signature of knowledge" of $\xi$. This rerandomized certificate and the signature of knowledge constitute the group signature.

The issuer should not know the random message $\xi$, because otherwise nonframeability is compromised. For this reason, in a group-joining protocol, the $\xi$ is jointly generated by the user and the issuer as follows: The user $i$ chooses a random exponent $\tau_{i}$ and sends $\tilde{r}=\tilde{x}^{\tau_{i}}$ to the issuer, while the issuer also chooses a random $\kappa_{i}$ and computes $\tilde{w}=\tilde{r} \cdot \tilde{x}^{\kappa_{i}}=\tilde{x}^{\tau_{i}+\kappa_{i}}$. This $\tau_{i}+\kappa_{i}$ will be used as the random message $\xi$ mentioned above. To establish a publicly verifiable 
connection between this $\xi$ and the user $i$, the user $i$ generates an (ordinary) signature on $k_{i}=e(g, \tilde{r})$ with a key pair which is previously registered in a public key infrastructure.

To open a signature, the opener uses $\tilde{w}$ to identify which $\xi$ is the message of the Camenisch-Lysyanskaya signature. Since $\tilde{w}$ makes the Camenisch-Lysyanskaya signature publicly verifiable, it cannot be used as an opening. Instead, the opener produces a non-interactive zero-knowledge proof of $\tilde{w}$ and $\kappa_{i}$ such that $k_{i}=$ $e(g, \tilde{w}) / e(g, \tilde{x})^{\kappa_{i}}$ and provides the signature on $k_{i}$. To verify this opening, a third party simply verifies the non-interactive zero-knowledge proof and the signature.

Unfortunately this scheme does not satisfy opening soundness. Assume a malicious signer obtains a group signature by an honest user, and further obtains an honestly generated opening of the signature. The proof of ownership contains $k_{i}$ and a signature on this by the honest user. The malicious signer replaces the signature on $k_{i}$ with his own signature on $k_{i}$. This forged opening passes the verification.

\section{$5 \quad$ Achieving Opening Soundness}

In this section we present a variant of the Groth scheme, which provides opening soundness (besides anonymity, non-frameability, and traceability).

\subsection{The Modified Groth Scheme}

The High-Level Idea. Let us first consider a general approach for achieving opening soundness.

The opener, who has the secret opening key, will always be able to determine the correct opening. To provide opening soundness, the opener needs to convince others that a given opening is correct. The easiest way to do that is to make the opening key public, but this will compromise the anonymity of the scheme. Instead, the opener can provide an NIZK proof of the correctness of an opening, to convince any third party. This is, in fact, the approach used in the BellareShi-Zhang construction.

If the opening algorithm essentially corresponds to a "decryption" of a ciphertext contained in the group signature (this is the case for many existing schemes), we might be able to take a different and more efficient approach. If the encryption scheme provides randomness recovering, the opener can simply release the randomness used for the ciphertext in question instead of an expensive zero-knowledge proof. Any third party will then be able to verify the correctness of an opening by re-encrypting the relevant information with the randomness provided by the opener, and then confirm that the resulting ciphertext is the same as the one contained in the signature.

In the Groth scheme, an opening essentially corresponds to the decryption of a linear encryption scheme. While linear encryption is not randomness-recovering, the opener is able to release related values which, together with an algebraic trick using a bilinear map, allow a third party to confirm that the decryption 
was done correctly. This property will allow us to add opening soundness to the original scheme. More specifically, in our variant of the Groth scheme, the opener, given a ciphertext $\left(c_{1}, c_{2}, c_{3}\right)=\left(F^{r}, H^{s}, v g^{r+s}\right)$, reveals $g^{r}$ and $g^{s}$ as a part of an opening. Using the properties of the bilinear map, these values can replace the exact randomness $r$ and $s$ when checking the correspondence between a ciphertext and a decryption: If a third party, given $g^{r}$ and $g^{s}$, wants to check the correspondence between a ciphertext $\left(c_{1}, c_{2}, c_{3}\right)$ and a decryption $v$, he simply checks whether the equations $e\left(F, g^{r}\right)=e\left(c_{1}, g\right),\left(H, g^{s}\right)=e\left(c_{2}, g\right)$, and $v=c_{3} /\left(g^{r} g^{s}\right)$ hold. If this is the case, he accepts the decryption as valid.

This idea is essentially the same as that used by Galindo et al. [15] in the context of public key encryption with non-interactive opening (PKENO). In [15, the application of PKENO schemes to group signature is briefly discussed as a mechanism for simplifying the construction of an opening. We will show that this technique is also able to ensure the opening soundness of group signature schemes.

Description of Our Variant. The Groth scheme can achieve opening soundness with the small modification shown in Fig. 2 ,

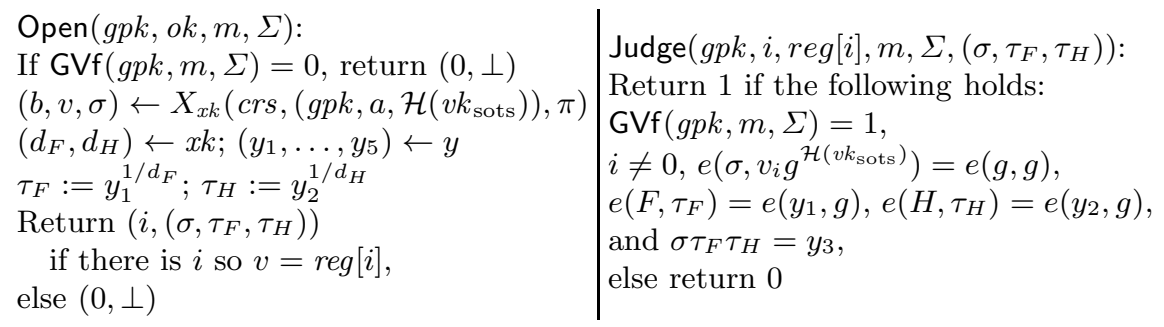

Fig. 2. The proposed modification of the Groth group signature scheme. The algorithms that do not appear in the figure are exactly the same as in Fig. 1

Theorem 2. The modified Groth scheme shown in Fig. 2 provides opening soundness.

Proof. Let us consider the game in Definition 6, and let $g p k$ be the group public key in the game, where the key is parsed to $(F, H, \cdots)$, and $\left(m, \Sigma, i, \tau, i^{\prime}, \tau^{\prime}\right)$ be the output of the adversary. Let $\Sigma, \tau$, and $\tau^{\prime}$ be parsed as follows: $\Sigma=$ $\left(v k_{\text {sots }}, a, \pi, y, \psi, \sigma_{\text {sots }}\right)$ in which $y=\left(y_{1}, y_{2}, y_{3}, y_{4}, y_{5}\right), \tau=\left(\sigma, \tau_{F}, \tau_{H}\right)$ and $\tau^{\prime}=$ $\left(\sigma^{\prime}, \tau_{F}^{\prime}, \tau_{H}^{\prime}\right)$.

We hereafter show that given a fixed $\Sigma$, it must hold that $i=i^{\prime}$ : Given a fixed $\Sigma$ (in particular $y_{1}, y_{2}$, and $y_{3}$ ), the verification equations

$$
e\left(F, \tau_{F}\right) \stackrel{?}{=} e\left(y_{1}, g\right) \wedge e\left(H, \tau_{H}\right) \stackrel{?}{=} e\left(y_{2}, g\right) \wedge \sigma \tau_{F} \tau_{H} \stackrel{?}{=} y_{3}
$$

uniquely determine $\tau_{F}, \tau_{H}$, and $\sigma$. Since both $\tau=\left(\sigma, \tau_{F}, \tau_{H}\right)$ and $\tau^{\prime}=\left(\sigma^{\prime}, \tau_{F}^{\prime}, \tau_{H}^{\prime}\right)$ pass the Judge verification, we must have that $\left(\sigma, \tau_{F}, \tau_{H}\right)=\left(\sigma^{\prime}, \tau_{F}^{\prime}, \tau_{H}^{\prime}\right)$. Now 
$v_{i}$ satisfies $e\left(\sigma, v_{i} g^{\mathcal{H}\left(v k_{\text {sots }}\right)}\right)=e(g, g)$ and $v_{i^{\prime}}$ satisfies $e\left(\sigma, v_{i^{\prime}} g^{\mathcal{H}\left(v k_{\text {sots }}\right)}\right)=e(g, g)$, but because $\sigma=\sigma^{\prime}$, and the equation $e\left(\sigma, v g^{\mathcal{H}\left(v k_{\text {sots }}\right)}\right)=e(g, g)$ uniquely determines $v$ given fixed $\sigma$ and $\mathcal{H}\left(v k_{\text {sots }}\right)$, we have that $v_{i}=v_{i^{\prime}}$, which implies that $i=i^{\prime}$.

The changes shown in Fig. 2 yields a scheme which is secure in the BSZ model i.e. the anonymity, the non-frameability, and the traceability of the original Groth scheme are maintained. This will be shown in the following.

Theorem 3. The modified Groth scheme provides anonymity if the decision linear assumption holds on $\mathbb{G}$, the one-time signature scheme is strongly unforgeable, and the hash function is target collision-resistant.

Proof (Sketch). The proof proceeds almost as in the original Groth scheme. The biggest difference from the original proof is that the simulator for the modified scheme needs to simulate two additional group elements $\left(\tau_{F}, \tau_{H}\right)=\left(g^{r}, g^{s}\right)$ when receiving an Open query. Note that in the simulation of Kiltz's tag-based encryption, when the simulator receives a decryption query $\left(y_{1}, y_{2}, y_{3}, y_{4}, y_{5}\right)=$ $\left(F^{r}, H^{s}, m g^{r+s},\left(g^{t} K\right)^{r},\left(g^{t} L\right)^{s}\right)$, the simulator at first extracts $g^{r}$ and $g^{s}$ without the knowledge of the decryption key and then simulates the decryption by computing $y_{3} / g^{r} g^{s}$. In a similar way, it is possible to simulate the two extra components required in our scheme.

Non-frameability and traceability can be proven more easily since these security notions do not require simulation of the Open oracle. For non-frameability, once an opening of the modified scheme that compromises the non-frameability notion is produced, one can obtain an opening for the original scheme (by simply dropping the extra components of $\tau_{F}$ and $\tau_{H}$ ) which will compromise the non-frameability of the original scheme.

Theorem 4. The modified Groth scheme provides non-frameability.

Theorem 5. The modified Groth scheme provides traceability.

\section{Conclusion}

We have identified an overlooked security concern for dynamic group signatures, namely, the possibility that a false opening proof can be produced by a corrupt user. To address this concern, we defined (two variants of) a new security notion denoted opening soundness, and furthermore discussed the opening soundness of several existing schemes. As a result, we have shown that the Bellare-Shi-Zhang construction 3 provides opening soundness as it is, and that small modifications to the Groth scheme (of the full version) [19] allow this scheme to provide opening soundness as well. We have also briefly discussed the opening soundness of some of the random oracle schemes [14/4, but leave further investigation of these schemes as future work. 
Acknowledgment. The authors would like to thank the anonymous reviewers of PKC 2012. The authors are also grateful to Benoît Libert for his invaluable comments.

\section{References}

1. Ateniese, G., Camenisch, J., Joye, M., Tsudik, G.: A Practical and Provably Secure Coalition-Resistant Group Signature Scheme. In: Bellare, M. (ed.) CRYPTO 2000. LNCS, vol. 1880, pp. 255-270. Springer, Heidelberg (2000)

2. Bellare, M., Micciancio, D., Warinschi, B.: Foundations of Group Signatures: Formal Definitions, Simplified Requirements, and a Construction Based on General Assumptions. In: Biham, E. (ed.) EUROCRYPT 2003. LNCS, vol. 2656, pp. 614-629. Springer, Heidelberg (2003)

3. Bellare, M., Shi, H., Zhang, C.: Foundations of Group Signatures: The Case of Dynamic Groups. In: Menezes, A. (ed.) CT-RSA 2005. LNCS, vol. 3376, pp. 136-153. Springer, Heidelberg (2005)

4. Bichsel, P., Camenisch, J., Neven, G., Smart, N.P., Warinschi, B.: Get Shorty via Group Signatures without Encryption. In: Garay, J.A., De Prisco, R. (eds.) SCN 2010. LNCS, vol. 6280, pp. 381-398. Springer, Heidelberg (2010)

5. Blum, M., De Santis, A., Micali, S., Persiano, G.: Noninteractive zero-knowledge. SIAM J. Comput. 20(6), 1084-1118 (1991)

6. Boneh, D., Boyen, X., Shacham, H.: Short Group Signatures. In: Franklin, M. (ed.) CRYPTO 2004. LNCS, vol. 3152, pp. 41-55. Springer, Heidelberg (2004)

7. Boyen, X., Waters, B.: Compact Group Signatures Without Random Oracles. In: Vaudenay, S. (ed.) EUROCRYPT 2006. LNCS, vol. 4004, pp. 427-444. Springer, Heidelberg (2006)

8. Boyen, X., Waters, B.: Full-Domain Subgroup Hiding and Constant-Size Group Signatures. In: Okamoto, T., Wang, X. (eds.) PKC 2007. LNCS, vol. 4450, pp. 1-15. Springer, Heidelberg (2007)

9. Camenisch, J., Lysyanskaya, A.: Signature Schemes and Anonymous Credentials from Bilinear Maps. In: Franklin, M. (ed.) CRYPTO 2004. LNCS, vol. 3152, pp. 56-72. Springer, Heidelberg (2004)

10. Camenisch, J., Michels, M.: A Group Signature Scheme with Improved Efficiency (Extended Abstract). In: Ohta, K., Pei, D. (eds.) ASIACRYPT 1998. LNCS, vol. 1514, pp. 160-174. Springer, Heidelberg (1998)

11. Chaum, D., van Heyst, E.: Group Signatures. In: Davies, D.W. (ed.) EUROCRYPT 1991. LNCS, vol. 547, pp. 257-265. Springer, Heidelberg (1991)

12. Delerablée, C., Pointcheval, D.: Dynamic Fully Anonymous Short Group Signatures. In: Nguyên, P.Q. (ed.) VIETCRYPT 2006. LNCS, vol. 4341, pp. 193-210. Springer, Heidelberg (2006)

13. Feige, U., Lapidot, D., Shamir, A.: Multiple non-interactive zero knowledge proofs based on a single random string. In: 31st Annual Symposium on Foundations of Computer Science, pp. 308-317. IEEE Computer Society (1990)

14. Furukawa, J., Imai, H.: An Efficient Group Signature Scheme from Bilinear Maps. In: Boyd, C., González Nieto, J.M. (eds.) ACISP 2005. LNCS, vol. 3574, pp. 455-467. Springer, Heidelberg (2005)

15. Galindo, D., Libert, B., Fischlin, M., Fuchsbauer, G., Lehmann, A., Manulis, M., Schröder, D.: Public-Key Encryption with Non-Interactive Opening: New Constructions and Stronger Definitions. In: Bernstein, D.J., Lange, T. (eds.) AFRICACRYPT 2010. LNCS, vol. 6055, pp. 333-350. Springer, Heidelberg (2010) 
16. Goldwasser, S., Micali, S., Rivest, R.L.: A digital signature scheme secure against adaptive chosen-message attacks. SIAM J. Comput. 17(2), 281-308 (1988)

17. Groth, J.: Simulation-Sound NIZK Proofs for a Practical Language and Constant Size Group Signatures. In: Lai, X., Chen, K. (eds.) ASIACRYPT 2006. LNCS, vol. 4284, pp. 444-459. Springer, Heidelberg (2006)

18. Groth, J.: Fully Anonymous Group Signatures Without Random Oracles. In: Kurosawa, K. (ed.) ASIACRYPT 2007. LNCS, vol. 4833, pp. 164-180. Springer, Heidelberg (2007)

19. Groth, J.: Fully anonymous group signatures without random oracles, May 17 (2010) (manuscript), http://www.cs.ucl.ac.uk/staff/J.Groth/CertiSignFull.pdf

20. Groth, J., Sahai, A.: Efficient Non-interactive Proof Systems for Bilinear Groups. In: Smart, N.P. (ed.) EUROCRYPT 2008. LNCS, vol. 4965, pp. 415-432. Springer, Heidelberg (2008)

21. Kiayias, A., Tsiounis, Y., Yung, M.: Traceable Signatures. In: Cachin, C., Camenisch, J.L. (eds.) EUROCRYPT 2004. LNCS, vol. 3027, pp. 571-589. Springer, Heidelberg (2004)

22. Kiayias, A., Yung, M.: Group Signatures with Efficient Concurrent Join. In: Cramer, R. (ed.) EUROCRYPT 2005. LNCS, vol. 3494, pp. 198-214. Springer, Heidelberg (2005)

23. Kilian, J., Petrank, E.: Identity Escrow. In: Krawczyk, H. (ed.) CRYPTO 1998. LNCS, vol. 1462, pp. 169-185. Springer, Heidelberg (1998)

24. Kiltz, E.: Chosen-Ciphertext Security from Tag-Based Encryption. In: Halevi, S., Rabin, T. (eds.) TCC 2006. LNCS, vol. 3876, pp. 581-600. Springer, Heidelberg (2006)

25. Rackoff, C., Simon, D.R.: Non-interactive Zero-Knowledge Proof of Knowledge and Chosen Ciphertext Attack. In: Feigenbaum, J. (ed.) CRYPTO 1991. LNCS, vol. 576, pp. 433-444. Springer, Heidelberg (1992)

26. Sahai, A.: Non-malleable non-interactive zero knowledge and adaptive chosenciphertext security. In: 40th Annual Symposium on Foundations of Computer Science, pp. 543-553. IEEE Computer Society (1999)

27. Zhou, S., Lin, D.: Shorter Verifier-Local Revocation Group Signatures from Bilinear Maps. In: Pointcheval, D., Mu, Y., Chen, K. (eds.) CANS 2006. LNCS, vol. 4301, pp. 126-143. Springer, Heidelberg (2006) 